\title{
Revealing the hopping mechanism of conduction in heavily doped silicon diodes
}

\author{
V.L. Borblik* , Yu.M. Shwarts, M.M. Shwarts \\ V. Lashkaryov Institute of Semiconductor Physics, NAS of Ukraine, \\ 41, prospect Nauky, 03028 Kyiv, Ukraine \\ Phone/Fax: +38 (044) 5257463 \\ *Phone: +38 (044) 525 6292, e-mail: borblik@lab2.semicond.kiev.ua
}

\begin{abstract}
Measurements of temperature dependences of excess tunnel current in heavily doped silicon $p-n$ junction diodes at fixed values of the forward bias are carried out in liquid helium temperature region. In some voltage interval, these dependences are described well by the Mott law for variable range hopping conductivity. The interpretation of these results considers a $p-n$ junction from a nontraditional point of view, namely, as heavily doped and highly compensated semiconductor.
\end{abstract}

Keywords: junction diode, temperature sensor, silicon, heavy doping, high compensation, hopping conductivity.

Manuscript received 18.04.05; accepted for publication 18.05.05.

\section{Introduction}

Silicon $p$ - $n$ junction diodes used as the temperature sensors have usually a sharp kink in their response curves in the temperature range where freezing-out of the current carriers occurs. In order to avoid this kink, we have developed and investigated silicon diodes where both sides of their $p-n$ junctions were doped up to the impurity concentrations providing metallic conductivity. In this case, the response curve holds quasi-linear character down to the liquid helium temperature [1]. In the region of such low temperatures, a current through the diode proves to be tunnel. Herewith, direct band-to-band tunneling is impossible, therefore we are talking here about the so-called excess tunnel current that is connected with presence of localized states in the forbidden semiconductor band.

This current is characterized by the dependence

$I=I_{0} \exp \left(U / U_{T}\right)$

where $U$ is the bias voltage, and $U_{T}$ is the temperatureindependent constant. The above dependence is believed to be connected with narrowing (under forward bias) the space charge region of the $p-n$ junction (see Ref. [2] and references therein). In the frame of such the approach, the factor $I_{0}$ is proportional to $\exp \left(-q V_{b i} / U_{T}\right)$, where $q$ is an electron charge, $V_{b i}$ is a built-in potential of the $p$ - $n$ junction. Its weak temperature dependence just determines the diode response curve in the range where tunnel current dominates. Under the degeneracy conditions $q V_{b i}=E_{g}(T)+\varepsilon_{F n}+\varepsilon_{F p}$ where $E_{g}(T)$ is the temperature-dependent energy gap of semiconductor, and $\varepsilon_{F n}, \varepsilon_{F p}$ are degeneracy degrees of electrons and holes, respectively. Therefore, temperature dependence of $V_{b i}$ is determined, mainly, by $E_{g}(T)$. Because $E_{g}(T)=E_{g}(0)-a T^{2} /(b+T)$ where $a$ and $b$ are the constants [3], at not low temperatures $E_{g}(T) \approx E_{g}(0)-c T$ and $I_{0} \propto \exp (A T)$. Such dependence was observed in the experiments so far [4]. We have studied the excess tunnel current at so low temperatures when $E_{g}(T) \approx E_{g}(0)=$ const and found as a result weak temperature dependence of $I_{0}$ satisfying the Mott law [5]. The obtained data have been interpreted by us on the basis of the electron theory of heavily doped semiconductors.

\section{Measurement results}

Measured current-voltage characteristics (CVC) of the heavily doped silicon $n^{++}-p^{+}$structure both sides of which possess metallic conductivity are represented in Fig. 1 (for the cryogenic temperature region). As was shown in Ref. [1], a visual criterion for domination of tunnel diode current in some range of temperatures and bias voltages is the quasi-parallel character of the CVCs (plotted in semilogarithmic scale) corresponding to different temperatures. In Fig. 1, this region extends from 1.0 to $1.08 \mathrm{~V}$ within the temperature range of $4.2-13.5 \mathrm{~K}$. 


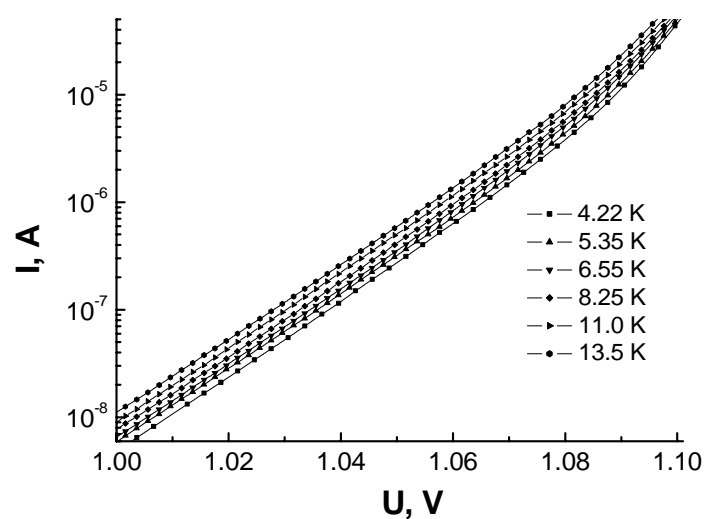

Fig. 1. Current-voltage characteristics of the silicon $n^{++}-p^{+}$-type diode in the cryogenic temperature region.

Shown in Fig. 2 are the temperature dependences of the diode current for a number of fixed values of the forward bias from the abovementioned interval. They are by no means described by $\exp (A T)$. These dependences plotted against reciprocal temperature (Fig. 3) give an evidence for variable activation energy of conductivity that is characteristic for variable range hopping conduction $[5,6]$. The same temperature dependences of the forward current (at the fixed bias voltages) plotted in the form which anticipates the Mott law [5] for conductivity

$$
I \propto \exp \left[-\left(T_{0} / T\right)^{1 / 4}\right]
$$

fall on straight lines satisfactorily in semi-logarithmic scale (Fig. 4). For greater persuasiveness, for one of the bias voltages $(U=1.02 \mathrm{~V})$, three temperature dependences of the diode current are integrated in Fig. 5: the first one is plotted as a function of reciprocal temperature, the second one - versus $(1 / T)^{1 / 2}$ (such dependences are also observed often under hopping

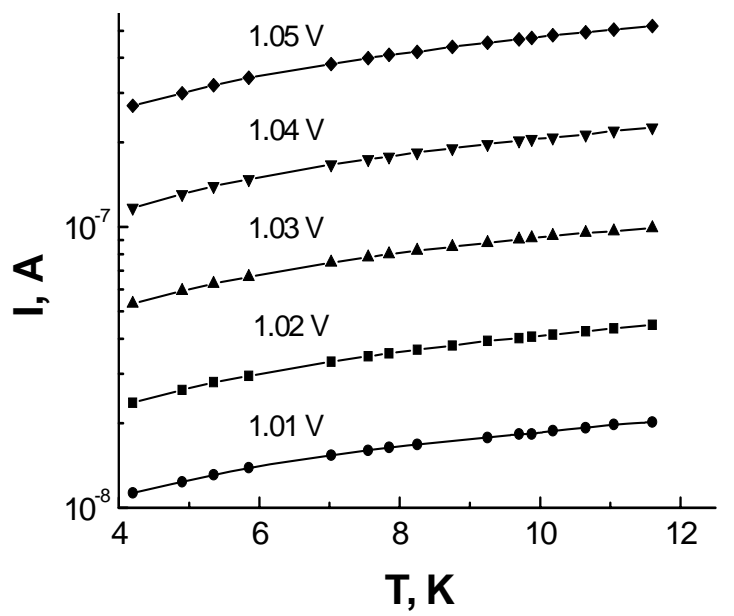

Fig. 2. Temperature dependences of the diode current at a number of bias voltages. conduction conditions), and the third one - against $(1 / T)^{1 / 4}$. Their comparison says persuasively in favor just of Mott's character of the temperature dependence for the diode current.

In our opinion, this fact firstly appears to be the first observation of Mott's conductivity in semiconductor structures with $p-n$ junctions, and, secondly, sheds additional light on the nature of the excess tunnel current in heavily doped junction diodes.

\section{Interpretation of the measurement results}

With a metallic character of the conductivity both in the emitter and in the base, the diode current is determined completely by resistance of the $p-n$ junction. The latter may be considered as heavily doped and highly compensated semiconductor. From this point of view, self-consistent spatial fluctuations of the electrostatic potential have to arise in the $p-n$ junction region which will bend both the bottom of the conduction band and the top of the valence one. As is known [6], the limiting scale of such fluctuations is available as to both magnitude and spatial extent.

From above their amplitude is limited by the bandgap value $E_{g}$ and their extent - by sizes of the order of $R_{g} \cong \frac{E_{g}^{2} \varepsilon^{2}}{N_{t} q^{4}}$ where $N_{t}=N_{D}+N_{A}$ is total concentration of donors and acceptors in the region of complete compensation, i.e. at metallurgical boundary of the $p-n$ junction, and $\varepsilon$ is dielectric constant of semiconductor. Let us compare this characteristic scale (i.e. $R_{g}$ ) with the width $w$ of the junction space charge region as it is was defined in a traditional theory of $p-n$ junctions [3]. Omitting the numerical coefficients (as well as it was made in the expression for $R_{g}$ ) we have

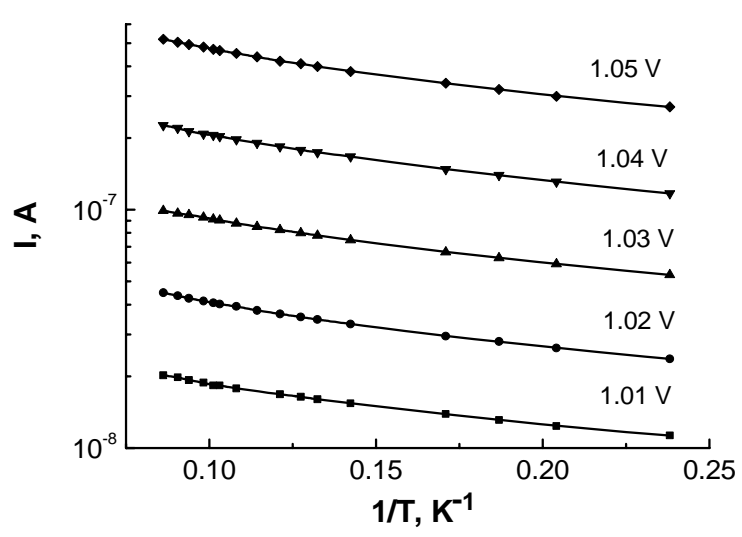

Fig. 3. Forward diode current versus reciprocal temperature at a number of voltage $U$ values. 


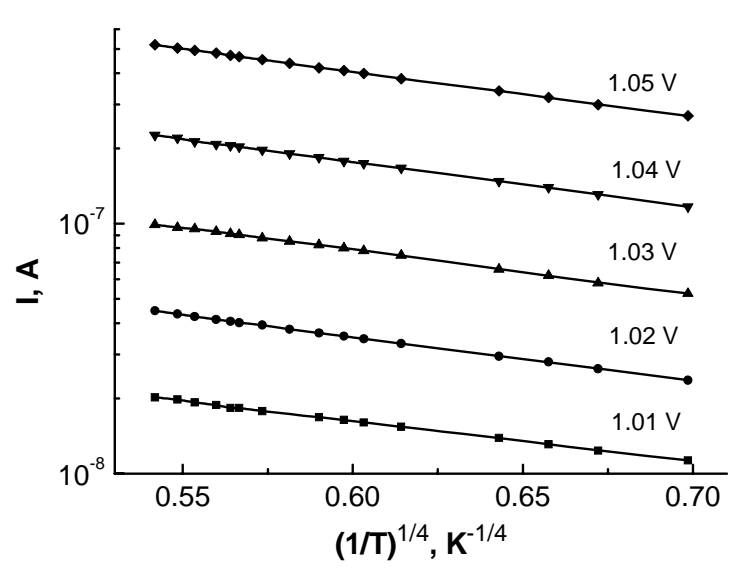

Fig. 4. Forward diode current at fixed voltage values as a function of $(1 / T)^{1 / 4}$.

$w \approx \sqrt{\frac{\varepsilon E_{g}}{N_{t} q^{2}}}$. The ratio $\frac{w^{2}}{R_{g}^{2}} \approx \frac{N_{t} q^{6}}{E_{g}^{3} \varepsilon^{3}}$ is proved to be of the order of unity if we take for estimations $N_{t} \sim 10^{18} \mathrm{~cm}^{-3}$, $E_{g} \sim 1 \mathrm{eV}, \varepsilon / \varepsilon_{0} \sim 10$ ( $\varepsilon_{0}$ is permittivity of vacuum). It is not surprising because both characteristic lengths are screening radii.

Thus, the so-called built-in potential of the $p-n$ junction may be considered as the most-large-scale electrostatic potential fluctuation. With deviation from the metallurgical boundary of the $p-n$ junction, the compensation degree will decrease, therefore the amplitude $\gamma$ of the large-scale potential fluctuations will reduce in accordance with the formula [7] $\gamma\left(\left|N_{D}-N_{A}\right|\right)=$ $=\frac{q^{2}}{\varepsilon} \frac{N_{m a j}^{2 / 3}}{\left|N_{D}-N_{A}\right|^{1 / 3}}$ where $N_{m a j}$ is concentration of the majority impurity. The characteristic scale $R_{C}$ of the large-scale fluctuations also reduces: $R_{C}\left(\left|N_{D}-N_{A}\right|\right)=$ $=\frac{N_{m a j}^{1 / 3}}{\left|N_{D}-N_{A}\right|^{2 / 3}}$.

Naturally, small-scale potential fluctuations will be superimposed in this large-scale picture according to the theory $[6,7]$. These small-scale fluctuations are limited from below by the size $R_{q}=\frac{a_{B}}{\left(N_{\text {maj }} a_{B}^{3}\right)^{1 / 9}}$ where $a_{B}$ is the Bohr radius of the bonded state of electron (or hole) at the single impurity center. Herewith, $R_{q}<a_{B}<<R_{g}$ because in the case under consideration $N_{m a j} a_{B}^{3}>>1$. Free current carriers come together in the wells of these minimal potential fluctuations forming "metallic drops". The obtained band picture is shown schematically in Fig. 6.

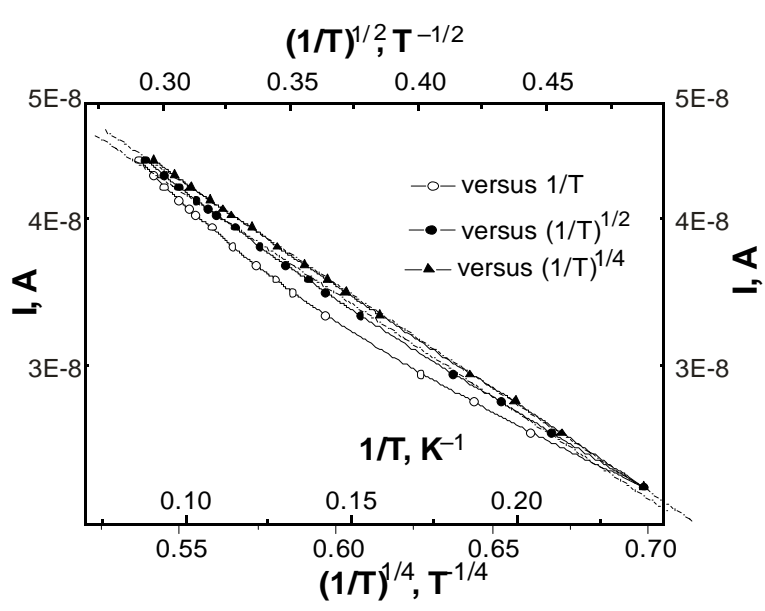

Fig. 5. Comparison of temperature dependences of the diode current (at $U=1.02 \mathrm{~V}$ ) plotted as a function of reciprocal temperature, and also versus $(1 / T)^{1 / 2}$ and against $(1 / T)^{1 / 4}$.

Under forward bias, the mechanism by means of which the current carriers overcome the main potential barrier remains the same as it was believed so far [2, 3] , i.e., tunneling through the intermediate localized states in the gap with subsequent recombination (paths A, B or C). But further, carriers have to pass through the "drops" systems at both sides of the $p-n$ junction. At low temperatures, such conduction will be hopping with the distinction that the hopes will be here not between localized states of carriers at the impurity centers (as usually) but between the "metallic drops" [7]. We believe that just this type of conduction is responsible for the Mott temperature dependence of the diode current observed by us, because under our conditions all current carriers have to be localized in the wells isolated one from another.
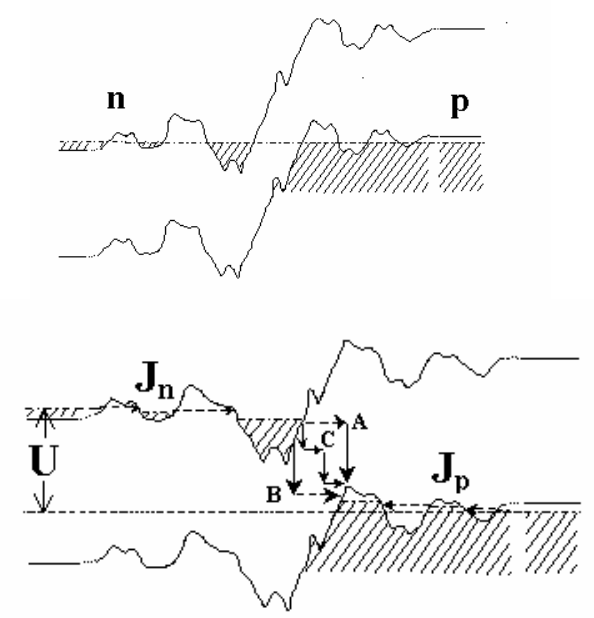

Fig. 6. Band scheme of a heavily doped $p-n$ junction in the bias absence (a) and under forward bias (b) with account of potential fluctuations (the filled states are shaded). 
It should be noted that it was the strong compensation $\left(N_{A} / N_{D} \approx 0.99\right)$ that had allowed to observe the variable range hopping conductivity in silicon for the first time [8]

Variable magnitude of the conductivity activation energy may be defined here as a derivative $-\frac{d(\ln I)}{d(k T)^{-1}}$. Corresponding analysis of the curves in Fig. 3 gives continuous series of this energy magnitudes decreasing from 0.55 to $0.27 \mathrm{meV}$ (for any bias voltage of considered interval). And a value of the parameter $T_{0}$ in (2) determined by analysis of the curves in Fig. 4 varies slightly with the voltage near the value of $T_{0}=300 \mathrm{~K}$.

In accordance with the theory of variable range hopping conduction [6], the average hopping length $\bar{R}$ is associated with a localization radius of current carriers by the dependence

$$
\bar{R} \approx a\left(\frac{T_{0}}{T}\right)^{1 / 4}
$$

which describes lengthening of the hop with temperature lowering. In the temperature range where we observe Mott's conductivity, the ratio $T_{0} / T$ varies from approximately 25 to 80 , and, consequently, the ratio $\bar{R} / a$ varies from 2.3 to 3 . Taking into consideration the evaluative character of the formula (3), one should believe that, in our case, average hopping length coincides, on the order of magnitude, with the localization radius for current carriers in anticipated drops.

\section{Conclusions}

So, the way of "linearization" of the thermodiode response curve by means of heavy doping the diode base undertaken by us in Ref. [1] gave really rise to desirable aim, but with some loss of sensitivity in the range of lowest temperatures. This fact is connected with domination in the diode current (under these conditions) of the tunnel current component lightly varying with temperature. However, its detailed investigation has allowed to us to reveal some fundamental properties of heavily doped diode structures. In particular, variable range hopping conductivity predicted for heavily doped and highly compensated (macroscopically homogeneous) semiconductors is found to be realized in heavily doped silicon $p-n$ junction diodes.

\section{References}

1. Yu.M. Shwarts, V.L. Borblik, N. R. Kulish, V.N. Sokolov, M.M. Shwarts, and E.F. Venger, Silicon diode temperature sensor without a kink of the response curve in cryogenic temperature region // Sensors and Actuators, 76(1-3), p. 107-111 (1999).

2. J.A. Del Alamo and R.N. Swanson, Forward-bias tunneling: a limitation to bipolar device scaling // IEEE Electron Device Letters, EDL-7(11), p. 629631 (1986).

3. S.M. Sze, Physics of Semiconductor Devices, $2^{\text {nd }}$ ed., John Wiley \& Sons, New York - Chichester - Brisbane - Toronto - Singapore (1981).

4. V.V. Yevstropov, Yu.V. Zhylyayev, M. Jumayeva, and N. Nazarov, Tunnel-excess current in nondegenerated barrier $p-n$ and $m-n$ structures $A^{\mathrm{III}} \mathrm{B}^{\mathrm{V}}$ on Si // Fiz. Tekh. Poluprov. 31(2), pp. 152-158 (1997).

5. N.F. Mott and E.A. Davis, Electron Processes in Non-crystalline Materials, $2^{\text {nd }}$ ed., Clarendon Press, Oxford (1979).

6. B.I. Shklovskii and A.L. Efros, Electron Properties of Doped Semiconductors, Springer, Berlin (1984).

7. B.I. Shklovskii, Hopping conduction of heavily doped semiconductors // Sov. Phys.-Semicond., 7(1), p. 77-83 (1973) [Fiz. Tekh. Poluprov. 7(1), p. 112$118(1973)]$.

8. M. Finetti, A.M. Mazzone, L. Passari, B. Ricco, and E. Susi, Temperature dependent conductivity of closely compensated phosphorus-doped silicon // Phil. Mag, 35(5), p. 1141-1151, (1977). 